\title{
Synthesis of new stable pseudobases
}

\author{
Zsuzsanna Riedl*, György Hajós, András Messmer, and Orsolya Egyed \\ Institute of Chemistry, Chemical Research Center, Hungarian Academy of Sciences, H-1525 \\ Budapest, POBox 17, Hungary \\ E-mail : zriedl@chemres.hu
}

Dedicated to Professor Branko Stanovnik on his $65^{\text {th }}$ birthday

(received 29 Aug 03; accepted 11 Nov 03; published on the web 26 Nov 03)

\begin{abstract}
Pyrido[1,2-b][1,2,4]triazinium salts easily form covalent hydrates in the presence of traces of water and readily react with various nucleophilic anions (e.g. hydoxide, alkoxide, or cyanide ions) to yield stable crystalline addition products (i.e. pseudobases). All these transformations were found to be regioselective and afforded 2-substituted products as revealed by NMR experiments.
\end{abstract}

Keywords: Fused 1,2,4-triazinium salt, pseudobase, covalent hydrate, regioselective addition

\section{Introduction}

Earlier we published ${ }^{1,2}$ that $\mathrm{N}$-amination of 2 -aminopyridines and a subsequent condensation reaction of the resulting diaminopyridinium salt with $\alpha$-dioxo reagents proved to be a simple and straightforward protocol for the synthesis of pyrido[1,2-b]-as-triazinium salts 1 (Scheme 1). We have also shown ${ }^{1}$ that this bicyclic heteroaromatic ring system is ready to form covalent hydrates 2. This water-sensibility of the heteroaromatic cation prompted us to reinvestigate the reactivity of $\mathbf{1}$ towards nucleophiles.

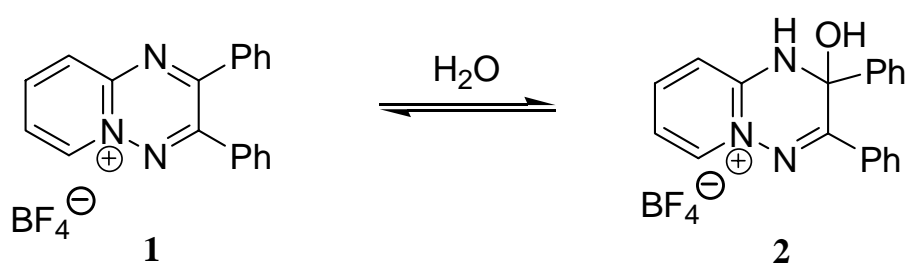

\section{Scheme 1}




\section{Results and Discussion}

We have found that the solution of 2,3-diphenylpyrido[1,2-b][1,2,4]triazinium fluoroborate $(5 a)^{3-5}$ in acetonitrile promptly reacts with aqueous sodium carbonate solution to give a pale yellow crystalline precipitate. NMR analysis of the product revealed that a stable pseudobase (7a) was formed, i.e. the hydroxide anion attacked the heteroaromatic system regioselectively in position 2 (Scheme 2).
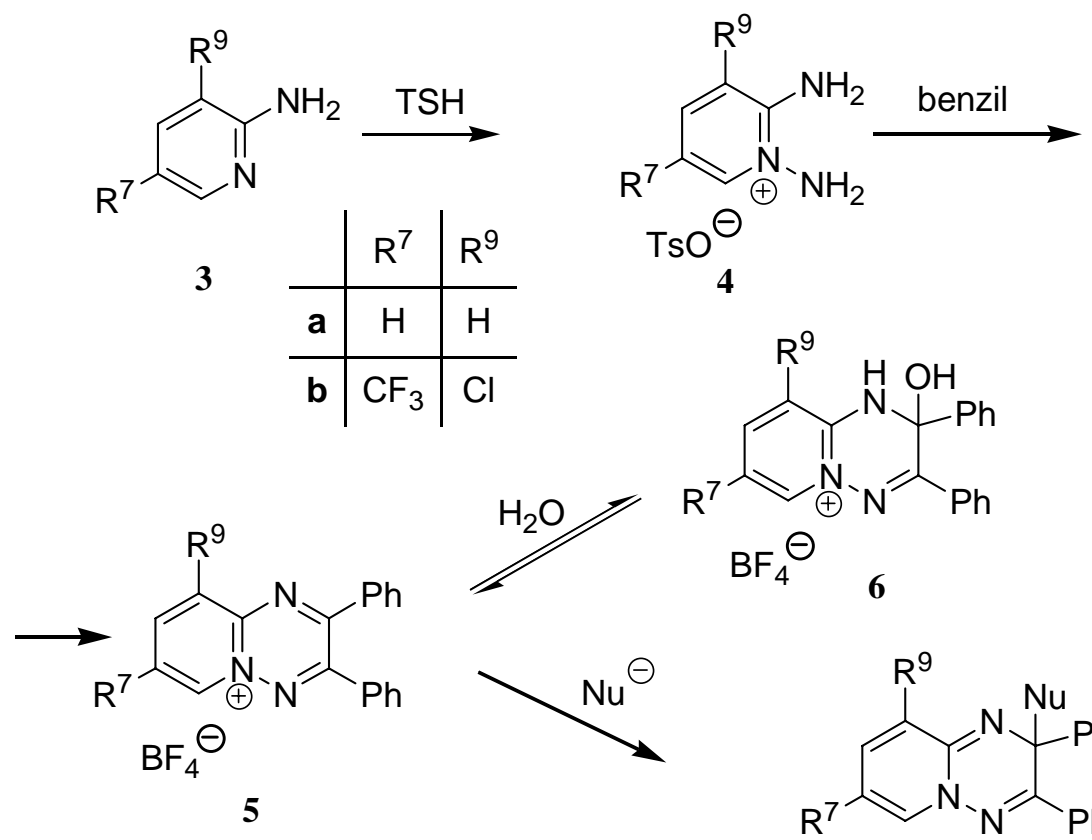<smiles>[R]C1=CN2N=C(c3ccccc3)C(N)(c3ccccc3)N=C2C([R])=C1</smiles>

\begin{tabular}{c|c|c|c|c} 
& $\mathrm{R}^{7}$ & $\mathrm{R}^{9}$ & $\mathrm{Nu}$ & $\ominus$ \\
\hline $\mathbf{a}$ & $\mathrm{H}$ & $\mathrm{H}$ & $\mathrm{OH}$ & 83 \\
\hline $\mathbf{b}$ & $\mathrm{H}$ & $\mathrm{H}$ & $\mathrm{OCH}_{3}$ & 90 \\
\hline $\mathbf{c}$ & $\mathrm{H}$ & $\mathrm{H}$ & $\mathrm{CN}$ & 72 \\
\hline $\mathbf{d}$ & $\mathrm{CF}_{3}$ & $\mathrm{Cl}$ & $\mathrm{OH}$ & 89 \\
\hline $\mathbf{e}$ & $\mathrm{CF}_{3}$ & $\mathrm{Cl}$ & $\mathrm{OCH}_{3}$ & 85 \\
\hline $\mathbf{f}$ & $\mathrm{CF}_{3}$ & $\mathrm{Cl}$ & $\mathrm{CN}$ & 73
\end{tabular}

\section{Scheme 2}

The solution of 5a in dimethyl sulfoxide at room temperature was found in accordance with our early observations ${ }^{1}$ ) to undergo a slow change as monitored by the NMR spectrum and after $24 \mathrm{~h}$ formation of the covalent hydrate $\mathbf{6 a}$ was detected. NMR experiments revealed that, similar 
to 7a, the hydroxyl group attached to the C-2 atom. Thus, the transformation $\mathbf{5 a} \rightarrow \mathbf{7 a}$ can be rationalized either as a nucleophilic addition of the hydroxide ion on $\mathbf{5 a}$ or as a rapid formation of $6 \mathbf{a}$ followed by a deprotonation step.

As both pathways are basically facilitated by the electron-defficient nature of the starting aromatic salt, introduction of further electron withdrawing groups seemed to assist such transformations. Upon this consideration we also have prepared derivative $\mathbf{5 b}$ containing an 9chloro and 7-trifluormethyl substituent and subjected both $\mathbf{5 a}$ and $\mathbf{5 b}$ to reactions with nucleophiles. Synthesis of $\mathbf{5 b}$ was carried out according to our well etablished procedure: 2amino-3-chloro-5-trifluoromethylpyridine (3) was $\mathrm{N}$-aminated by tosyl hydroxylamine to the diamino salt 4 and reaction with benzil yielded the desired fused triazinium salt.

The new pyrido[1,2-b][1,2,4]triazinium salt (5b) also easily reacted with sodium carbonate solution and gave the expected addition product 7d in good yield. Similar reactions of $\mathbf{5 a}$ and $\mathbf{5 b}$ were experienced also with sodium methoxide and sodium cyanide to give the addition products 7b,c and 7e,f, respectively.

Proton assignment for both $\mathbf{7 b}$ and $\mathbf{7 e}$ was fulfilled by decoupling and homonuclear correlation experiments. The carbons were assigned with heterocorrelated methods. HSQC measurements supplied the one-bond proton-carbon connectivities, while quaternary carbons were determined by applying the HMBC method. In the case of $\mathbf{7 b}$ the protons and the corresponding carbons at positions $6,7,8$, and 9 could be determined staightforwardly. The protons and carbons of the two phenyl groups were distinguished on the basis of their long range heterocorrelations. The carbon at $84.7 \mathrm{ppm}$ gave crosspeaks with both protons $\mathrm{H}-2+\mathrm{H} 6$ at 7.36 ppm and with the methoxy protons $(3.18 \mathrm{ppm})$. These correlations were diagnostic from the point of view of the methoxy position at C-2 instead of at C-9a. The situation was rather similar for compound $7 \mathbf{e}$.

Preliminary experiments indicated that similar to the nucleophilic anions, also secondary amines can react with triazinium salt 5 . In this case, however, a different course of the reaction was also observed in some cases ${ }^{6,7}$ (i.e. ring opening of the pyridine moiety to afford dieneamines). Investigation of this ambident reactivity towards amines is in progress and will be published elsewhere. Also, because of the potential biological use from the viewpoint of pharmaceutical applications, various tests are planned.

\section{Conclusions}

These results indicate that the pyrido[1,2-b][1,2,4]triazinium salts (5) are reactive towards nucleophilic reagents in position 2. This reactivity allowed the selective synthesis of 2substituted pseudobases containing hydroxide, methoxide, and cyano groups. HMBC experiments unambiguously revealed the position of these substituents. 


\section{Experimental Section}

General Procedures. Melting points were determined by a Büchi apparatus and are uncorrected. The IR spectra were recorded with a Nicolet Magna 750 FT-IR, spectrophotometers; the ${ }^{1} \mathrm{H}$ and ${ }^{13} \mathrm{C}$ NMR spectra were recorded using a Varain UNITY-INOVA spectrometer, equipped with a 5 -mm indirect detection probe, operating at $399.9 \mathrm{MHz}$ for ${ }^{1} \mathrm{H}$ and at $100.5 \mathrm{MHz}$ for ${ }^{13} \mathrm{C}$ nuclei. Approximately $20 \mathrm{mg}$ of each sample was dissolved in $0.65 \mathrm{ml} \mathrm{CDCl}_{3}$ or DMSO-d $\mathrm{d}_{6}$. The temperature was stabilized at $303 \mathrm{~K}$. All $1 \mathrm{D}$ and $2 \mathrm{D}$ experiments were run using the Varian software library. Assignments of the phenyl-protons and carbons were marked by 2'-H, 3'-H, 2'$\mathrm{C}$, etc. in the case of the 2-phenyl group, and 2"-H, 3"-H, 2"-C, etc. with the 3-phenyl group.

1,2-Diamino-3-chloro-5-(trifluoromethyl)pyridinium tosylate (4b). To a solution of 2-amino3-chloro-5-trifluoromethylpyridine $(3,1.96 \mathrm{~g}, 10 \mathrm{mmol})$ in dichloromethane $(20 \mathrm{ml})$ was added a solution of tosyl hydroxylamine ${ }^{8}(2.0 \mathrm{~g}, 10.7 \mathrm{mmol})$ in dichloromethane $(40 \mathrm{ml})$ was added at room temperature whereupon a yellow solid precipitated. After $1 \mathrm{~h}$ diethyl ether $(30 \mathrm{ml})$ was added and the preicipitate was filtered off and recrystallized from methanol/diethyl ether to give colorless crystals, $3.37 \mathrm{~g}(0.88 \mathrm{mmol}, 88 \%)$, mp 209-211 ${ }^{\circ} \mathrm{C}$. IR (KBr): 3266, 3131, 3027, 1666, $1335,1189,1172,1136,1126,1035,1012,904,819,687 \mathrm{~cm}^{-1} ;{ }^{1} \mathrm{H}$ NMR $\delta\left(\right.$ DMSO-d $\left._{6}\right): 2.28(\mathrm{~s}$, $3 \mathrm{H}, \mathrm{H}-\mathrm{CH}_{3}$ ), 7.05 (s, 2H, H-NH${ }_{2}$ ) 7.10, 7.47 (m, 4H, H-p-tolyl)), 8.57 (s, 1H, H-4), 8.69 (s, 1H, $\mathrm{H}-6), 9.29 \mathrm{~s},\left(2 \mathrm{H}, \mathrm{H}-\mathrm{NH}_{2}\right)$; Anal. Calcd. for $\mathrm{C}_{13} \mathrm{H}_{13} \mathrm{ClF}_{3} \mathrm{~N}_{3} \mathrm{O}_{3} \mathrm{~S}$ (383.77): C, 40.69; H, 3.41; N, 10.95. Found: C, 40.53; H, 3.54; N, 10.85 .

2,3-Diphenyl-pyrido[1,2-b][1,2,4]triazin-5-ium tetrafluoroborate (5a). This compound was prepared according to literature procedure $\mathrm{s}^{3-5}$ by addition of tetrafluoroboric acid, mp $255-257^{\circ} \mathrm{C}$. IR (KBr): 3105, 1630, 1582, 1563, 1395, 1292, 1083, 1031, 784, $696 \mathrm{~cm}^{-1} ;{ }^{1} \mathrm{H}$ NMR $\delta($ $\mathrm{CDCl}_{3}+\mathrm{TFA}$ ): 7.40-7.50 (m, 4H, H-3', H-5', H-3", H-5"), 7.60 (m, 4H, H-2', H-4', H-6', H-4"), 7.67 (m, 2H, H-2", H-6"), 8.14 (ddd, 1H, $J=7.0,6.5,1.5 \mathrm{~Hz}, \mathrm{H}-7$ ), 8.50 (dd, $1 \mathrm{H}, J=7.9,1.5 \mathrm{~Hz}$, $\mathrm{H}-9$ ), 8.63 (ddd, $1 \mathrm{H}, J=7.9,7.0,1.0 \mathrm{~Hz}, \mathrm{H}-8), 9.18$ (dd, $1 \mathrm{H}, J=6.5,1.5 \mathrm{~Hz}, \mathrm{H}-6$ ); ${ }^{13} \mathrm{C}$ NMR $\delta$ ( $\mathrm{CDCl}_{3}$, TFA): 126.6 (C-7), 128.4 (C-9), 129.2, 129.4 (C-3', C-5', C-3", C-5"), 130.0 (C-2', C6"), 130.9 (C-2", C-6"), 131.3, 133.2 (C-1', C-1"), 132.7 (C-4'), 134.0 (C-4"), 139.8 (C-6), 144.5 (C-8), 145.1 (C-9a), 157.1 (C-2), 161.2 (C-3). Anal. Calcd. for $\mathrm{C}_{19} \mathrm{H}_{14} \mathrm{BF}_{4} \mathrm{~N}_{3}$ (371.14): C, 61.49; H, 3.80; N, 11.32. Found: C, 61.53; H, 3.79; N, 11.05 .

2-Hydroxy-2,3-diphenyl-1,2-dihydro-pyrido[1,2-b][1,2,4]triazin-5-ium tetrafluoroborate (6a). Formation of this compound was observed when a solution of 5a in dimethyl sulfoxide was allowed to stand at room temperature for $24 \mathrm{~h} .{ }^{1} \mathrm{H}$ NMR $\delta\left(\right.$ DMSO-d $\left._{6}\right): 7.15$ (ddd, $1 \mathrm{H}, J=7.0$, 6.5, 1.5 Hz, H-7), 7.20 (dd, 1H, J = 9, 1.5 Hz, H-9), 7.26 - 7.38 (m, 4H, H-3', H-5', H-3", H-5"), 7.45, 7.55 (m, 2H, H-4', H-4"), 7.60 (m, 2H, H-2', H-6'), 7,71 (m, 2H, H-2", H-6"), 8.01 (ddd, $1 \mathrm{H}, J=9,7,1.5 \mathrm{~Hz}, \mathrm{H}-8), 8.53(\mathrm{dd}, 1 \mathrm{H}, J=6.5,1.5 \mathrm{~Hz}, \mathrm{H}-6), 8.84(\mathrm{~s}, 1 \mathrm{H}, \mathrm{NH}) ;{ }^{13} \mathrm{C}$ NMR $\delta$ (DMSO-d ${ }_{6}$ ): 75.8 (C-2), 114.7, 114.8 (C-7, C-9), 127.6 (C-2', C-6'), 129.0 (C-3', C-5', C-3", C5"), 129.9 (C-2", C-6"), 132.0, 132.2 (C-4', C-4"), 134.4, 141.5 (C-1', C-1"), 138.6 (C-6), 143.7 (C-8), 144.0 (C-9a), 158.4 (C-3). 
9-Chloro-2,3-Diphenyl-7-trifluoromethyl-pyrido[1,2-b][1,2,4]triazin-5-ium fluoroborate (5b). A mixture of $\mathbf{4 b}(1 \mathrm{~g}, 2.6 \mathrm{mmol})$, benzil $(0.6 \mathrm{~g}, 2.85 \mathrm{mmol})$ and sulfuric acid $(10 \mathrm{ml})$ was let to stand in a closed flask at room temperature for $48 \mathrm{~h}$. The resulting reddish yellow solution was poured onto crushed ice (100 g) and, then, $40 \%$ tetrafluoroboric acid (2 ml) was added. A yellow preicipitate was formed which was filtered off and recrystallized from nitromethane/diethyl ether. Yield: $0.85 \mathrm{~g}$ (1.8 mmol, 68\%), mp 233-242 ${ }^{\circ} \mathrm{C}$. IR (KBr): 3150 , 1644, 1559, 1430, 1377, 1282, 1171, 1084, 1054, 774, $704 \mathrm{~cm}^{-1} ;{ }^{1} \mathrm{H} \mathrm{NMR} \delta\left(\mathrm{CDCl}_{3}+\mathrm{TFA}\right)$ : 7.39 (m, 2H, H-3", H-5"), 7.43 (m, 2H, H-3', H-5'), 7.59 (m, 2H, H-2', H-6'), 7.60 (m, 1H, H-4'), 7.62 (m, 1H, H-4"), 7.76 (m, 2H, H-2", H-6"), 8.59 (s, 1H, H-8), 9.27 (s, 1H, H-6); ${ }^{13} \mathrm{C}$ NMR $\delta$ $\left(\mathrm{CDCl}_{3}+\mathrm{TFA}\right): 120.8\left(\mathrm{q},{ }^{1} J_{C, F}=265 \mathrm{~Hz}, \mathrm{CF}_{3}\right), 127.9$ (q, $\left.{ }^{2} J_{C, F}=36 \mathrm{~Hz}, \mathrm{C}-7\right), 129.3,129.4\left(\mathrm{C}-3^{\prime}, \mathrm{C}-\right.$ 5', C-3", C-5"), 130.1 (C-2', C-6'), 130.9 , 132.9 (C-1', C-1"), 131.8 (C-2", C-6"), 133.0 (C-4'), 135.1 (C-4"), 135.7 (C-9), 136.7 (q, $\left.{ }^{3} J_{C, F}=4 \mathrm{~Hz}, \mathrm{C}-8\right), 138.3$ (q, $\left.{ }^{3} J_{C, F}=4 \mathrm{~Hz}, \mathrm{C}-6\right), 145.0$ (C9a), 158.0 (C-2), 163.3 (C-3). Anal. Calcd. for $\mathrm{C}_{20} \mathrm{H}_{12} \mathrm{BClF}_{7} \mathrm{~N}_{3}$ (473.58): C, 50.72; H, 2.55; N, 8.87. Found: C, 51.03; H, 2.57; N, 8.45.

9-Chloro-2-hydroxy-2,3-diphenyl-7-trifluoromethyl-1,2-dihydro-pyrido[1,2-b][1,2,4]triazin-

5-ium tetrafluoroborate (6b). Formation of this compound was observed when a solution of $\mathbf{5 b}$ in dimethyl sulfoxide was allowed to stand at room temperature for $24 \mathrm{~h} .{ }^{1} \mathrm{H}$ NMR $\delta\left(\mathrm{DMSO}-\mathrm{d}_{6}\right)$ : 7.20-7.40 (m, 6H, H-3', H-4', H-5', H-3", H-4", H-5"), 7.55 (m, 2H, H-2', H-6'), 7.74 (m, 2H, H2", H-6"), 8.53 (s, 1H, H-8), 9.12 (s, 1H, H-6); ${ }^{13} \mathrm{C}$ NMR $\delta$ (DMSO-d $): 78.0$ (C-2), 122.3 (q, $\left.{ }^{1} J_{C, F}=265 \mathrm{~Hz}, \mathrm{CF}_{3}\right), 127.6\left(\mathrm{C}-2^{\prime}, \mathrm{C}-6^{\prime}\right), 128.7,128.9\left(\mathrm{C}-3^{\prime}, \mathrm{C}-5^{\prime}, \mathrm{C}-3{ }^{\prime \prime}, \mathrm{C}-5^{\prime \prime}\right), 129.7$ (q, ${ }^{2} J_{C, F}=$ $35 \mathrm{~Hz}, \mathrm{C}-7), 130.1$ (C-2", C-6"), 131.7 (C-9), 133.7, 134.0 (C-1', C-1"), 135.3 (q, ${ }^{3} J_{C, F}=5 \mathrm{~Hz}$, C-8), $137.5\left(\mathrm{q},{ }^{3} J_{C, F}=5 \mathrm{~Hz}, \mathrm{C}-6\right), 142.8(\mathrm{C}-9 \mathrm{a}), 157.4(\mathrm{C}-3)$.

\section{General procedure for the synthesis of pseudobases 7 from pyrido[1,2-b][1,2,4]triazinium} salts 5

To a suspension of $5 \mathbf{a}(0.37 \mathrm{~g}, 1 \mathrm{mmol})$ or $5 \mathbf{b}(0.47 \mathrm{~g}, 1 \mathrm{mmol})$ in acetonitrile $(2 \mathrm{ml})$ was added the appropriate reagent (i.e. aqueous sodium carbonate solution, methanolic sodium methoxide, or aqueous potassium cyanide, $3 \mathrm{mmol}$ ) with stirring at $5{ }^{\circ} \mathrm{C}$. A yellow solution was formed from which the product separated either spontaneously or by addition of ice. The product was filtered off and recrystallized from cyclohexane.

2,3-Diphenyl-2H-pyrido[1,2-b][1,2,4]triazine-2-ol (7a). Yield: 0.25 g (0.83 mmol, 83 \%), mp 146-152 ${ }^{\circ} \mathrm{C}$. IR (KBr): 3027, 2797, 1650, 1558, 1541, 1444, 1329, 1282, 1178, 1157, 1137 , 1066, 777, 747, $703 \mathrm{~cm}^{-1} ;{ }^{1} \mathrm{H}$ NMR $\delta\left(\mathrm{DMSO}_{-} \mathrm{d}_{6}\right): 6.07$ (t, 1H, $\left.J=6.7, \mathrm{~Hz}, \mathrm{H}-7\right), 6.45$ (d, 1H, $J$ $=9 \mathrm{~Hz}, \mathrm{H}-9), 6.89$ (s, 1H, H-OH), 7.03 (ddd, 1H, $J=9,6.7,1.5 \mathrm{~Hz}, \mathrm{H}-8), 7.40-7.10$ (m, 8H, H3', H-5', H-2', H-6', H-4', H-3", H-4", H-5"), 7.72 (m, 3H, H-6, H-2",.H-6"); ${ }^{13} \mathrm{C}$ NMR $\delta($ DMSO-d 6 ): 78.5 (C-2), 104.5 (C-7), 122.1 (C-9), 125.6 (C-2', C-6'), 126.8 (C-4'), 127.7 (C-3', C5', C-3", C-5"), 129.3 (C-2", C-6", C-4"), 133.8 (C-8), 134.5, 143.1 (C-1', C-1"), 135.9 (C-6), 147.3 (C-3), 145.8 (C-9a). Anal. Calcd. for $\mathrm{C}_{19} \mathrm{H}_{15} \mathrm{~N}_{3} \mathrm{O}$ (301.34): C, 75.73; H, 5.02; N, 13.94. Found: C, 75.58; H, 5.09; N, 13.90 . 
2-Methoxy-2,3-diphenyl-2H-pyrido[1,2-b][1,2,4]triazine (7b). Yield: $0.28 \mathrm{~g}$ (0.90 mmol, 90 \%), mp 127-133 ${ }^{\circ} \mathrm{C}$. IR (KBr): 3079, 3058, 2976, 2943, 2820, 1651, 1550, 1537, 1444, 1238, 1036, 1019, 940, 771, 757, 694, $598 \mathrm{~cm}^{-1} ;{ }^{1} \mathrm{H}$ NMR $\delta\left(\mathrm{CDCl}_{3}+\mathrm{DMSO}_{6}\right): 3.18$ (s, 3H, H-OMe), 6.10 (ddd, 1H, $J=7.2,6.9,1.2 \mathrm{~Hz}, \mathrm{H}-7$ ), 6.55 (dd, 1H, $J=9,1.8 \mathrm{~Hz}, \mathrm{H}-9$ ), 7.04 (ddd, 1H, $J=9$, 7.2, 1.8 Hz, H-8), 7.15 (m, 1H, H-4'), 7.20-7.30 (m, 5H, H-3', H-5', H-3", H-4", H-5"), 7.36 (m, 2H, H-2', H-6'), 7.63 (dd, 1H, $J=6.9,1.8 \mathrm{~Hz}, \mathrm{H}-6), 7.76$ (m, 2H, H-2", H-6"); ${ }^{13} \mathrm{C}$ NMR $\delta$ $\left(\mathrm{CDCl}_{3}+\mathrm{DMSO}_{6}\right): 50.5(\mathrm{O}-\mathrm{Me}), 84.7$ (C-2), 105.6 (C-7), 122.6 (C-9), 126.2 (C-2', C-6'), 127.7 (C-4'), 128.4 (C-3', C-5', C-3", C-5"), 129.3 (C-2", C-6"), 130.2 (C-4"), 134.5, 146.5 (C-1', C-1"), 134.7 (C-8), 136.3 (C-6), 145.8 (C-9a), 147.1 (C-3). Anal. Calcd. for $\mathrm{C}_{20} \mathrm{H}_{17} \mathrm{~N}_{3} \mathrm{O}$ (315.37): C, 76.17; H, 5.43; N, 13.32. Found: C, 75.98; H, 5.30; N, 13.05.

2,3-Diphenyl-2H-pyrido[1,2-b][1,2,4]triazine-2-carbonitrile (7c). Yield: $0.22 \mathrm{~g}(0.72 \mathrm{mmol}$, $72 \%$ ), mp 128-130 ${ }^{\circ} \mathrm{C}$. IR (KBr): 3087, 3060, 3040, 2924, 2243, 1645, 1548, 1534, 1447, 1332, 1238, 1148, 1129, 758, 692, $557 \mathrm{~cm}^{-1} ;{ }^{1} \mathrm{H}$ NMR $\delta\left(\mathrm{DMSO}_{-} \mathrm{d}_{6}+\mathrm{CDCl}_{3}\right): 6.12(\mathrm{dd}, 1 \mathrm{H}, J=6.8$, $1.4 \mathrm{~Hz}, \mathrm{H}-7), 6.49$ (d, 1H, $J=9.5 \mathrm{~Hz}, \mathrm{H}-9), 7.05$ (ddd, 1H, $J=9.5,6.8,1.6 \mathrm{~Hz}, \mathrm{H}-8), 7.15$ (m, 1H,), 7.60 - 7.30 (m, 10 H, H-2', H-3', H-4', H-5', H-6', H-2", H-3", H-4", H-5", H-6"), 7.65 (d, $1 \mathrm{H}, J=6.8, \mathrm{~Hz}, \mathrm{H}-6) ;{ }^{13} \mathrm{C} \mathrm{NMR} \delta\left(\mathrm{DMSO}_{-} \mathrm{d}_{6}+\mathrm{CDCl}_{3}\right): 59.3,105.7,118.5,121.4,125.4128 .3$, $128.5,129.1,129.4,131.1,131.9,135.1,135.9$, 139.6, 141.7, 144.4. Anal. Calcd. for $\mathrm{C}_{20} \mathrm{H}_{14} \mathrm{~N}_{4}$ (310.35): C, 77.40; H, 4.55; N, 18.05. Found: C, 77.28; H, 4.47; N, 17.90.

9-Chloro-2,3-diphenyl-7-trifluoromethyl-2H-pyrido[1,2-b][1,2,4]triazine-2-ol (7d). Yield: 0.36 g (0.89 mmol, $89 \%$ ), mp 139-142 ${ }^{\circ} \mathrm{C}$. IR (KBr): 3258, 3016, 3085, 3070, 3052, 1661, $1591,1315,1166,1126,1070,1003,893,771,708,688 \mathrm{~cm}^{-1} ;{ }^{1} \mathrm{H}$ NMR $\delta\left(\mathrm{DMSO}_{6}\right): 7.12(\mathrm{~m}$, 1H, H-4'), 7.15-7.46 (m, 7H, H-2', H-3', H-6', H-5', H-3", H-4", H-5"), 7.75 (d, J = 2 Hz, H-8), 7.80 (m, 2H, H-2", H-6"), 8.41 (m, 1H, H-6); ${ }^{13} \mathrm{C} \mathrm{NMR} \delta$ ( $\left.\mathrm{CDCl}_{3}, \mathrm{DMSO}\right): 78.8$ (C-2), 105.3, 126.1, 126.7, 127.4, 127.8, 129.4, 129.9, 133.5, 133.2, 135.7, 139.5, 145.9 , 151.8. Anal. Calcd. for $\mathrm{C}_{20} \mathrm{H}_{13} \mathrm{ClF}_{3} \mathrm{~N}_{3} \mathrm{O}$ (403.78): C, 59.49; H, 3.25; N, 10.41. Found: C, 59.38; H, 3.11; N, 10.20.

2-Methoxy-9-chloro-2,3-diphenyl-7-trifluoromethyl-pyrido[1,2-b][1,2,4]triazine (7e). Yield: $0.35 \mathrm{~g}$ (0.85 mmol, $85 \%)$, mp 180-183 ${ }^{\circ} \mathrm{C}$. IR (KBr): 3110, 3083, 3059, 2941, 2819, 1665, 1616, 1600, 1314, 1165, 1127, 1078, $1046869,770,703,687 \mathrm{~cm}^{-1} ;{ }^{1} \mathrm{H}_{\mathrm{NMR}} \delta\left(\mathrm{CDCl}_{3}+\right.$ DMSO-d $\left.)_{6}\right): 3.21$ (s, 3H, OMe), 7.22 (m, 1H, H-4'), 7.28-7.30 (m, 4H, H-3', H-5', H-3", H-5"), 7.36 (m, 1H, H-4"), 7.41 (m, 2H, H-2', H-6'), 7.55 (d, J=2 Hz, H-8), 7.80 (m, 2H, H-2", H-6"), $8.24(\mathrm{~m}, 1 \mathrm{H}, \mathrm{H}-6) ;{ }^{13} \mathrm{C}$ NMR $\delta\left(\mathrm{CDCl}_{3}+\mathrm{DMSO}_{\mathrm{d}}\right): 51.2(\mathrm{OMe}), 85.0(\mathrm{C}-2), 107.0\left(\mathrm{q},{ }^{2} J_{C, F}=\right.$ $35 \mathrm{~Hz}, \mathrm{C}-7), 123.3$ (q, ${ }^{1} J_{C, F}=270 \mathrm{~Hz}, \mathrm{CF}_{3}$ ), 126.2 (C-2', C-6'), 127.0 (C-9), 128.4 (C-8), 128.6, 128.7 (C-3', C-5', C-3", C-5"), 129.6 (C-2", C-6"), 131.1 (C-4"), 133.2, 145.1 (C-1', C-1"), 135.8 (q, $\left.{ }^{3} J_{C, F}=5 \mathrm{~Hz}, \mathrm{C}-6\right), 141.9$ (C-9a), 150.2 (C-3). Anal. Calcd. for $\mathrm{C}_{21} \mathrm{H}_{15} \mathrm{ClF}_{3} \mathrm{~N}_{3} \mathrm{O}$ (417.81): C, 60.37; H, 3.62; N, 10.06. Found: C, 60.08; H, 3.57; N, 9.85.

\section{9-Chloro-2,3-diphenyl-7-trifluoromethyl-2H-pyrido[1,2-b][1,2,4]triazine-2-carbonitrile (7f).}

Yield: $0.30 \mathrm{~g}$ (0.73 mmol, 73 \%),mp 195-200 ${ }^{\circ} \mathrm{C}$. IR (KBr): 3108, 3060, 2925, 2185, 1664, $1622,1599,1419,1316,1164,1145,1113,868,765,700 \mathrm{~cm}^{-1} ;{ }^{1} \mathrm{H}$ NMR $\delta\left(\mathrm{CDCl}_{3}\right): 7.20-7.50$ (m, 8H, H-2', H-3', H-6', H-4', H-5', H-3", H-4", H-5"), 7.61 (m, 2H, H-2", H-6"), 7.68 (m, 1H, $\mathrm{H}-8), 7.86(\mathrm{~m}, 1 \mathrm{H}, \mathrm{H}-6) ;{ }^{13} \mathrm{C} \mathrm{NMR} \delta\left(\mathrm{CDCl}_{3}\right): 60.4$ (C-2), 108.4, 118.0, 126.3, 128.2, 128.6, 
127.8, 129.1, 129.7, 131.4, 132.0, 134.1, 138.8, 141.3 , 146.1. Anal. Calcd. for $\mathrm{C}_{21} \mathrm{H}_{12} \mathrm{ClF}_{3} \mathrm{~N}_{4}$ (412.79): C, 61.10; H, 2.93; N, 13.57. Found: C, 61.09; H, 2.97; N, 13.28.

\section{Acknowledgments}

Thanks are due to financial support provided by research funds OTKA T 33105, T 35176 as well as $1 / 047$ NKFP MediChem.

\section{References}

1. Hajós, Gy.; Riedl, Zs.; G-Baitz, E.; Messmer, A. Tetrahedron 1992, 48, 8459.

2. Hajós, Gy.; Messmer, A.; Bátori, S.; Riedl, Zs. Bull. Soc. Chim. Belg. 1992, 101, 597.

3. Hajós, Gy.; Riedl, Zs.; Messmer, A. Acta Chem. Scand. 1993, 47, 296.

4. Baranova, N. V.; Sheinkman, A. K.; Kost, A. N. Khim. Get. Soed. 1970, 1148.

5. Baranova, N. V.; Sheinkman, A. K.; Kost, A. N. Khim. Get. Soed. 1973, 1266.

6. Kotschy, A.; Hajós, Gy.; Messmer, A. J. Org. Chem. 1995, 60, 4919.

7. Hajós, Gy.; Kotschy, A. Acta Chim. Slov. 1998, 45, 285.

8. Glover, E. E.; Rowbottom, K. T. J. Chem. Soc., Perk. Trans. I 1976, 367. 